\title{
Research Paper \\ The Relationship Between Spiritual Health and Successful Aging
}

\author{
Fatemeh Yousefi ${ }^{1}$, ${ }^{*}$ Fatemeh Mohammadi ${ }^{2}$, Seyedeh Ameneh Motalebi ${ }^{3}\left(\mathbb{C}\right.$, Saeed Pahlevan Sharif ${ }^{\circledR}$
}

1. Department of Nursing, School of Nursing and Midwifery, Qazvin University of Medical Sciences, Qazvin, Iran

2. Social Determinants of Health Research Center, Qazvin University of Medical Sciences, Qazvin, Iran.

3. School of Taylor's Business, Taylor's University, Selangor, Malaysia.

Clteat on: Yousefi F, Mohammadi F, Motalebi SA, Pahlevan Sharif S. [The Relationship Between Spiritual Health and Successful Aging (Persian)]. Iranian Journal of Ageing. 2020; 15(2):246-257. https://doi.org/10.32598/sija.2020.3.2183.2

doi' https://doi.org/10.32598/sija.2020.3.2183.2

Received: 20 Jul 2019

Accepted: 08 Dec 2019

Available Online: 01 July 2020

\section{ABSTRACT}

Objectives Successful aging is a positive inner feeling and satisfaction with the past and present life experiences. Thus, the present study aimed to determine the predicting factors of successful aging in a sample of Iranian elderly.

Methods \& Materials This cross-sectional and descriptive-analytic study was conducted on 291 elderly in Qazvin City, Iran, in 2018. The study participants were selected using the two-stage cluster sampling method. Data collection was performed using the demographic characteristics checklist, the Successful Aging Scale, and Paloutzian and Ellison Spiritual Wellbeing Scale. Data analysis was conducted using descriptive tests (frequency, percentage, central \& dispersion indices) and a hierarchical linear regression model.

Results The study sample was 291 elderly with a Mean \pm SD age of $68.3 \pm 6.7$ years. The obtained results indicated that the spiritual health of $81.1 \%$ of the explored elderly was strong; most of the examined elderly presented a good level of successful aging. Besides, spiritual health was a predictor of successful aging in the study participants. Regarding the aspects of spiritual health, existential health positively and significantly impacted successful aging $(B=0.65, P<0.001)$. Furthermore, the obtained data revealed that the employed elderly (B:4.29), compared to housewives (B:-4.25), those with high economic status, compared to those with a moderate (B:-3.44) and low (B:-7.332) economic level, and the elderly without a history of physical (B:-4.55) and mental (B:-2.54) conditions obtained significantly higher scores in successful aging.

Conclusion The existential aspect of spiritual health is among the predictors of successful aging. Given the importance and sensitivity of the issues related to the elderly, by interventions focused on spiritual education, one can increase the quality of this period and enrich it.

\section{Extended Abstract}

\section{Introduction}

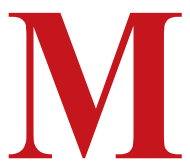

edical advances and improved health levels have led to an increase in life expectancy [1], followed by a dramatic increase in the global elderly population. and continues in the $21^{\text {st }}$ century, as the aging century of the world's population [2]. The number of the elderly in 2000 was approximately equal to 600 million worldwide and it is estimated that the elderly will shape $>20 \%$ of the world's population by 2050 [3]. According to the United Nations Office, the population of the elderly aged $\geq 60$ years in Iran will increase from about $8.2 \%$ in 2015 to $14.4 \%$ in 2030 and $31.2 \%$ in 2050 [4]. Spiritual health in old age is positively associated with various criteria of life satisfaction, modulates

Such a population inflammation initiated in the 20th century

\section{* Corresponding Author:}

Fatemeh Mohammadi, PhD.

Address: Social Determinants of Health Research Center, Qazvin University of Medical Sciences, Qazvin, Iran.

Tel: +98 (912) 1824233

E-mail: mohammadi1508@gmail.com 
biopsychological health, and is beneficial in search of meaning and purpose in life; it presents a significant impact on mental health and success in this period [16]. Research has indicated that spirituality increases in old age. For example, Wink and Dillon (2002) conducted a longitudinal study and concluded that the level of spiritual health significantly increases from mid to late-life [17]. Studies suggested that spiritual forces in the elderly and patients lead to indescribable peace, strength, and vitality. Besides, the elderly patients with higher religious and spiritual beliefs encounter fewer cognitive problems $[18,19]$. Iran is also experiencing the transition to an aging population and the number of elderly is on the rise. Therefore, there is a significant need for research to identify the predictors of successful aging concerning social policy. Moreover, due to limited studies on successful aging and spiritual health, the present study aimed to determine the predictability of spiritual health for successful aging.

\section{Materials and Methods}

The present descriptive-analytical study was conducted on the elderly in Qazvin City, Iran, in 2018. According to the research by Goli et al. (2016) entitled "the relationship between successful aging and spiritual health in the elderly in Tehran", the sample size was determined by considering $95 \%$ confidence level $(\alpha=0.05)$ as 228 subjects. The final estimate of 290 subjects considered per $30 \%$ probability of non-response. Cluster sampling was performed in two stages; first, Qazvin City was divided into 5 regions (clusters), then, eligible samples were selected by convenience sampling method from each cluster. Initially, a list of public centers (e.g., mosques, parks, \& daycare centers for the elderly) was prepared and a mosque and a park were selected from each cluster. Two daycare centers accepting members from all parts of the city were considered for sampling. The inclusion criteria of the study were the age of $\geq 60$ years, the orien- tation of time, place, and person, ability to communicate verbally, and willingness to participate in the study. Moreover, presenting psychological conditions (Alzheimer's disease \& major depression disorder), difficulty in communicating, severe audiovisual impairments, and debilitating diseases included the exclusion criteria of the study. The required data were collected using a demographic information checklist, the Successful Aging Scale (SAS), and Paloutzian and Ellison Spiritual Wellbeing Scale.

\section{Results}

The study sample consisted of 291 elderlies with a Mean \pm SD age of $68.3 \pm 6.7$ years and an age range of $60-87$ years. In total, $162(57.7 \%)$ of the study samples were men. Most of the research participants were in the age group of $60-70$ years $(63.9 \%, 186$ subjects $)$, married $(73.9 \%, 215$ individuals), had $\geq 5$ children ( $41.6 \%, 121$ subjects), and lived with their spouse or children $(70.4 \%, 205$ individuals). The source of income of most of them was themselves $(34.4 \%$, $\mathrm{n}=100)$; the income level of most of them was moderate (42.3\%, $n=123)$; approximately one-third of the study subjects were illiterate $(43 \%, n=121)$, and the majority of them were housewives or retired $(35.7 \%, n=104)$. Furthermore, almost most of the study participants presented no history of hospitalization $(54.6 \%, \mathrm{n}=159)$. Regarding the history of physical illnesses, the majority of the study subjects reported a history of physical illness $(81.1 \%, \mathrm{n}=236)$ and only $5.8 \%$ $(n=17)$ reported mental health illnesses. As per Table 1, the spiritual health of most of the examined elderly $(81.1 \%)$ was at a strong level and their Mean \pm SD level of spiritual health equaled $87.68 \pm 15.37$. The results of stepwise regression analysis are demonstrated in Table 2 . In simultaneous regression analysis, all independent variables were entered into the analysis; beta values indicated the significance of the independent variables in predicting the regression equation. The collected results suggested that the independent variables en-

Table 1. The levels of spiritual health in the explored elderly

\begin{tabular}{|c|c|c|c|}
\hline Spiritual Health & & No. (\%) & \\
\hline Low & & $2(0.7)$ & \\
\hline Moderate & & $53(18.2)$ & \\
\hline High & & $236(81.1)$ & \\
\hline Total & & $291(100.0)$ & \\
\hline Spiritual health & Mean $\pm S D$ & & Range \\
\hline $20-120$ & $87.68 \pm 15.37$ & & $22-119$ \\
\hline
\end{tabular}


Table 2. Predicting successful aging based on the dimensions of spiritual health and demographic characteristics

\begin{tabular}{|c|c|c|c|c|c|c|c|}
\hline \multirow{2}{*}{\multicolumn{2}{|c|}{ Characteristic }} & \multicolumn{2}{|c|}{ Unstandardized Coefficients } & \multirow{2}{*}{$\boldsymbol{\beta}$} & \multirow{2}{*}{$\mathbf{P}$} & \multicolumn{2}{|c|}{$95 \% \mathrm{Cl}$} \\
\hline & & $\beta$ & SD & & & Lower Bound & Upper Bound \\
\hline \multicolumn{2}{|c|}{ Constant } & 70.315 & 3.11 & & 0.000 & 63.011 & 77.6197 \\
\hline \multicolumn{2}{|c|}{ Existential health } & 0.658 & 0.078 & 0.390 & 0.000 & 0.505 & 0.811 \\
\hline Occupation & $\begin{array}{l}\text { Housekeeper } \\
\text { Employed }\end{array}$ & $\begin{array}{c}-4.254 \\
4.291\end{array}$ & $\begin{array}{l}1.339 \\
1.889\end{array}$ & $\begin{array}{l}-1.51 \\
0.110\end{array}$ & $\begin{array}{l}0.002 \\
0.024\end{array}$ & $\begin{array}{l}-6.890 \\
0.572\end{array}$ & $\begin{array}{l}-1.618 \\
8.010\end{array}$ \\
\hline Income level & $\begin{array}{l}\text { Low } \\
\text { Moderate }\end{array}$ & $\begin{array}{l}-7.332 \\
-3.444\end{array}$ & $\begin{array}{l}1.780 \\
1.676\end{array}$ & $\begin{array}{l}-0.260 \\
-0.126\end{array}$ & $\begin{array}{l}0.000 \\
0.041\end{array}$ & $\begin{array}{l}-10.837 \\
-6.743\end{array}$ & $\begin{array}{l}-3.828 \\
-0.144\end{array}$ \\
\hline \multicolumn{2}{|c|}{ Mental health disorders (no) } & -2.541 & 2.707 & -0.217 & 0.000 & -17.870 & -7.213 \\
\hline \multicolumn{2}{|c|}{ A history of physical illnesses (no) } & 4.554 & 1.590 & 0.132 & 0.004 & 1.425 & 7.683 \\
\hline
\end{tabular}

Model summary; $\mathrm{R}=0.633, \mathrm{R}^{2}=0.440$, Adjusted $\mathrm{R}^{2}=0.424$. SE: Standard Error, CI: Confidence Interval. SA A M A N D

tered in multivariate regression analysis could predict up to $44 \%$ of successful aging in the study participants.

\section{Discussion and Conclusion}

Successful aging refers to the acquisition of individual potential and the optimal level of biopsychosocial ability through which the elderly feel satisfied with life. A characteristic that may be associated with successful aging is spiritual health [3]. This study aimed to determine the role of spiritual health in predicting successful aging. The present study results indicated that the spiritual health of $81.1 \%$ of the explored elderly was at a strong level. This finding was in line with those of most previous studies [22, 23]. The obtained data revealed a positive and significant relationship between spiritual health and successful aging. In another study, Islamic spirituality training positively influenced successful aging. In other words, the average score of successful aging in the intervention group was higher than that in the controls [25]. Additionally, the current research findings signified that improved religiosity and spiritual health declined the odds of mental distress and the feelings of despair and negative attitude on late-life stages in the examined elderly $[26,27]$. Besides, the subjects who believed in the afterlife did not find life meaningless and hollow and were satisfied with their lives [28]. Moreover, spirituality plays a critical role in accepting crises; according to social nursing, human is a multidimensional being with the dimension of spirituality at the center [9].

The current study results revealed a significant relationship between spiritual health and successful aging. Besides, the existential health dimension was recognized as a predictor of successful aging. Therefore, to have successful aging for completing the final stages of lives with better health and op- timal psychosocial functions, providing spiritual education programs and improving existential health (through managing psychosocial concerns and improving the relationship between the individual, society, \& environment) can be effective. It is hoped that health policymakers take effective practical measures to improve spirituality in the elderly.

\section{Ethical Considerations}

\section{Compliance with ethical guidelines}

All ethical principles are considered in this article. The participants were informed about the purpose of the research and its implementation stages. They were also assured about the confidentiality of their information and were free to leave the study whenever they wished, and if desired, the research results would be available to them.

\section{Funding}

This research did not receive any grant from funding agencies in the public, commercial, or non-profit sectors.

\section{Authors' contributions}

All authors equally contributed to preparing this article.

\section{Conflicts of interest}

The authors declared no conflict of interest.

\section{Acknowledgements}

The authors would like to thank the Vice Chancellor for Research of Qazvin University of Medical Sciences. 


\title{
آيا سلامت معنوى سالمندى موفق را بيشگَويى كند؟
}

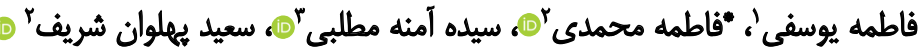

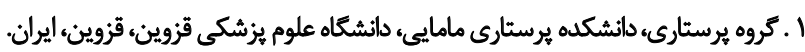

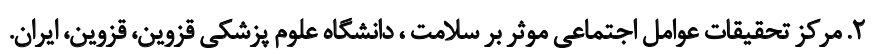

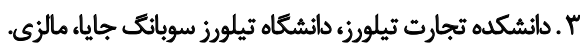

\begin{abstract}
حكSי

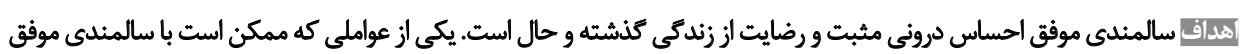

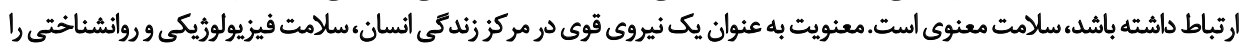

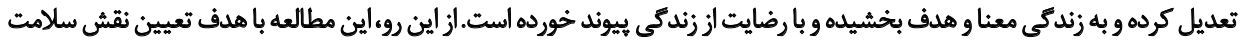

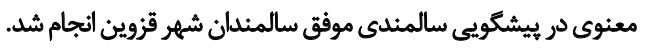

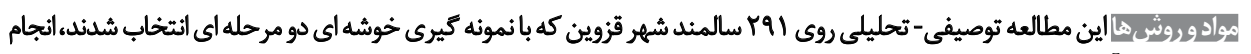

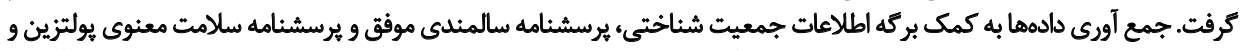

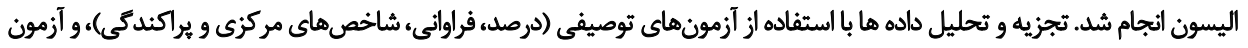

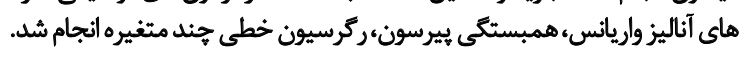

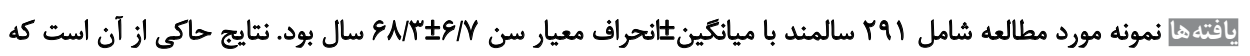

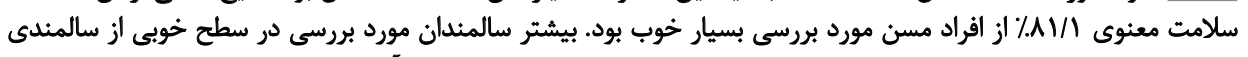

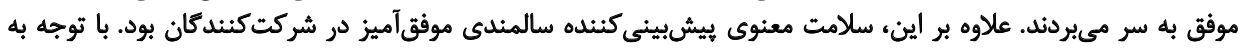

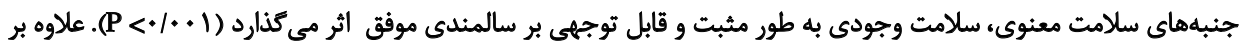

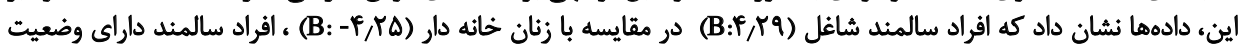

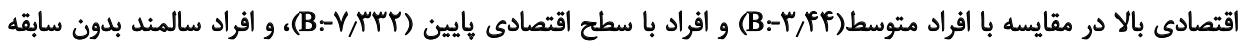
شرايط جسمى(B)

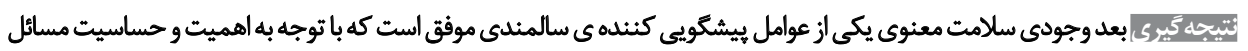

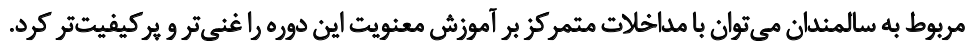

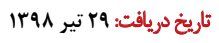

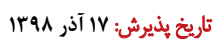

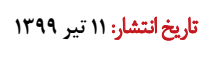

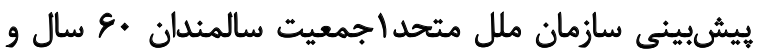

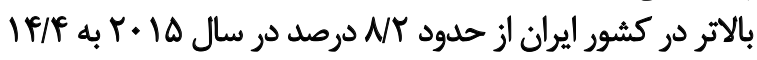

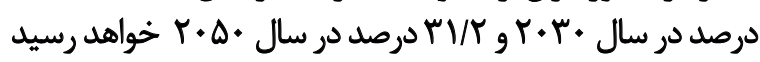

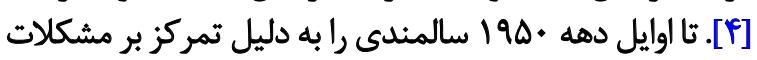

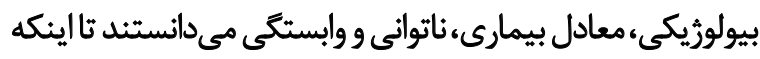

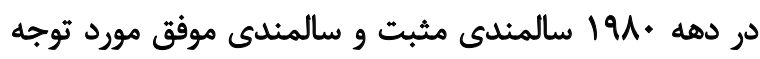

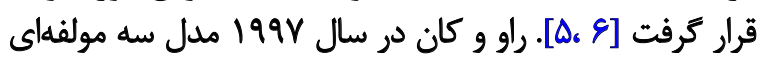

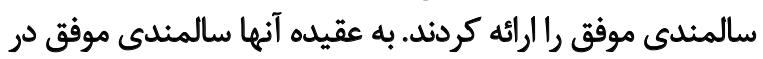
بر كيرنده "احتمال بايين وجود بيمارى"، "كنش بالائ ذئل ذهنى ييشرفتهاى يزشكى و بالا رفتن سطح بهداشت منجر به

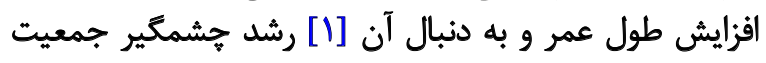

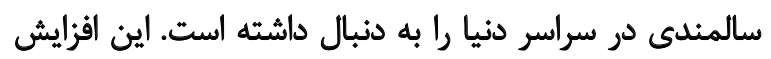

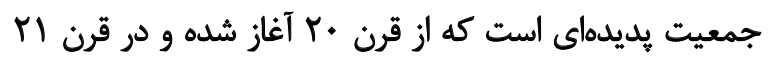

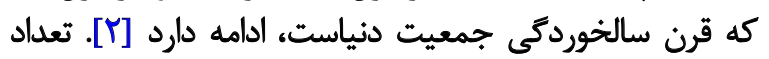

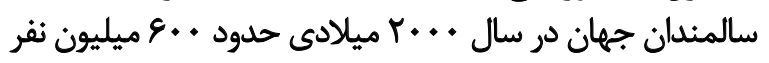

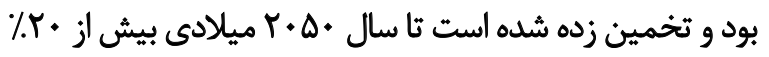

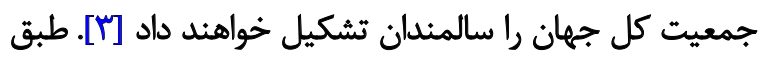

1. United Nation

قويسئده مسئول:

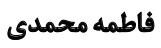

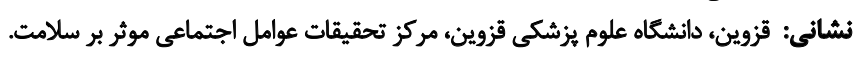

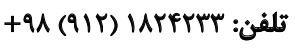
بـ إن الكترونيكي: mohammadi1508@gmail.com 
سالخوردكّى جمعيت است و بطور روز افزون بر تعداد سالمندان

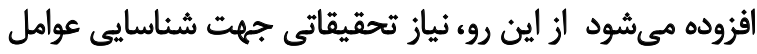

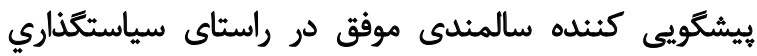

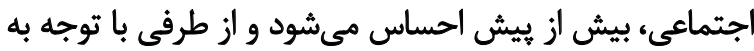

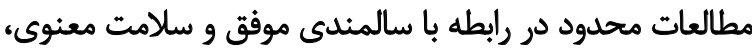

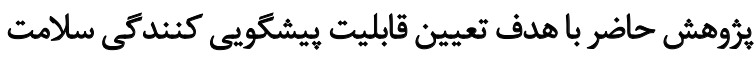
معنوى براى سالمندى موفق انجام كرفت

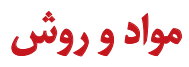

اين ثرُوهش يك مطالعه توصيفى - تحليلى است كه در سال كاي

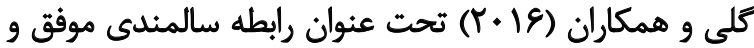

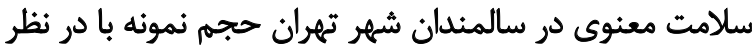

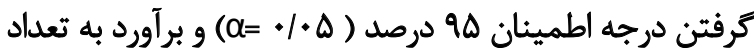

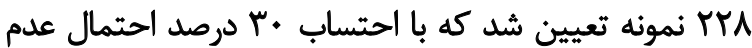

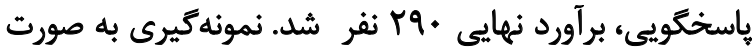

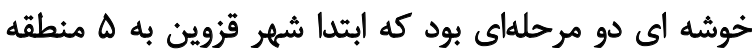

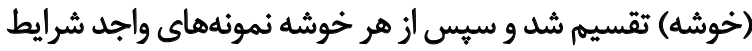

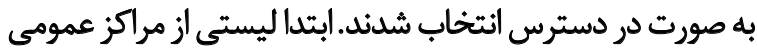

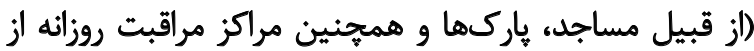

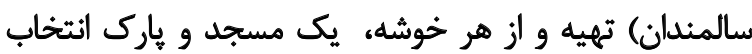

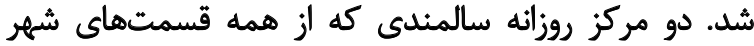

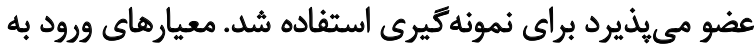

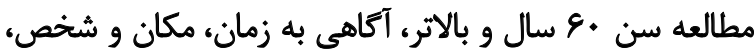

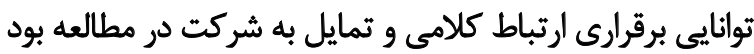

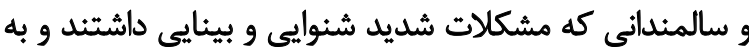

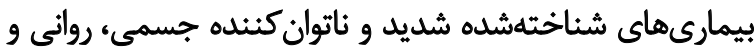

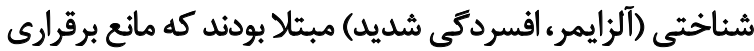

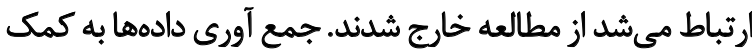

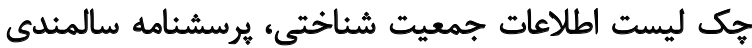

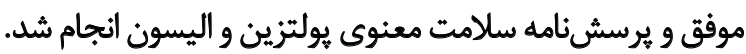

يرسشنامه اطلاعات دموكرافيك شامل اطلاعاتى در رابطه با

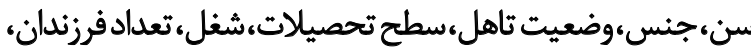

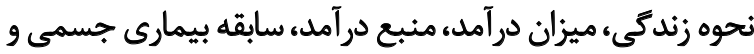

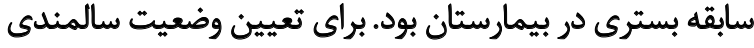

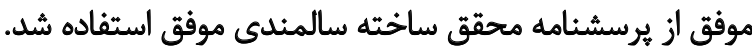

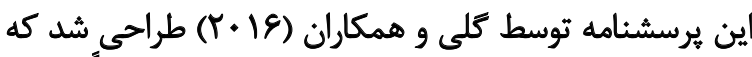

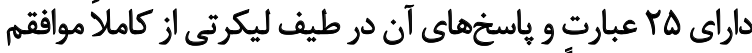

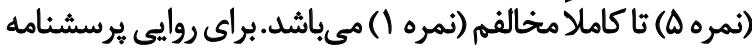

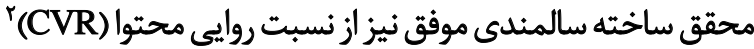

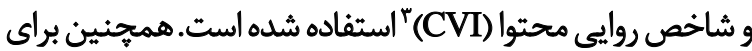

2. Content Validity Ratio

3. Content Validity Index

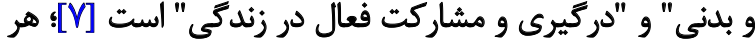

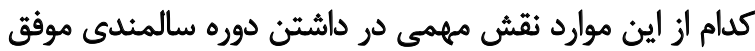

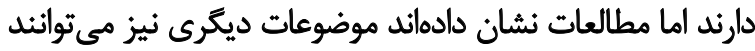

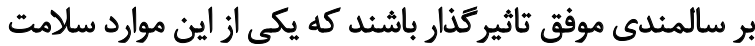

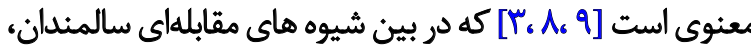

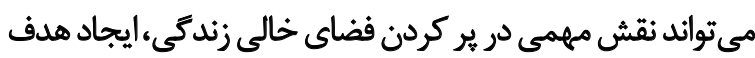

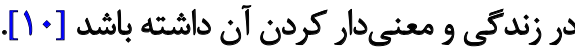

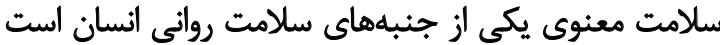

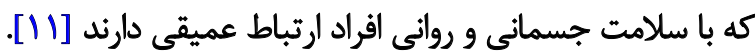

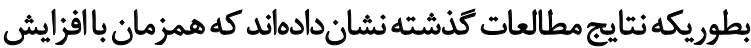

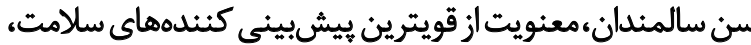

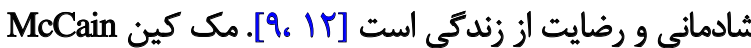

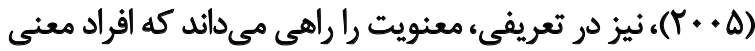

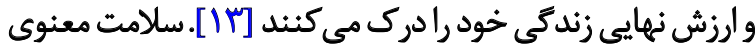

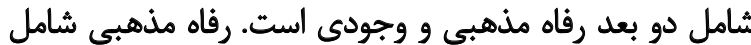

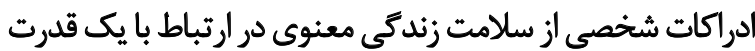

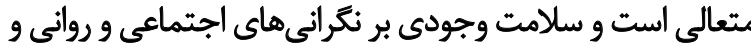

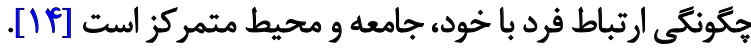

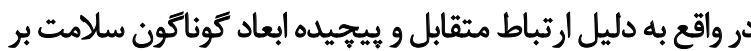

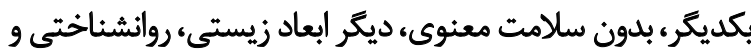

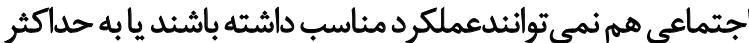

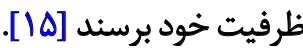

سلامت معنوى در دوران سالمندى روابط مثبت با معيارهاى

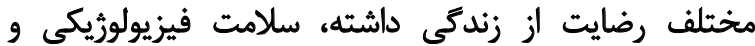

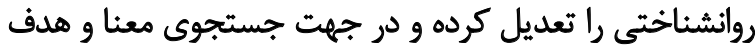

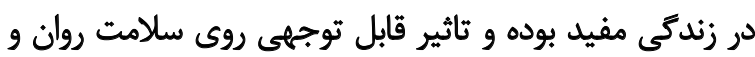

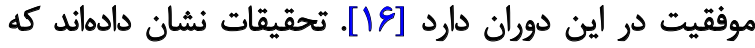

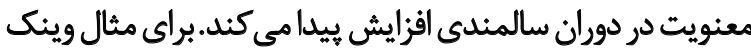

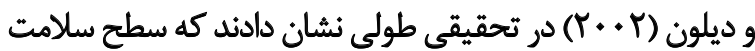

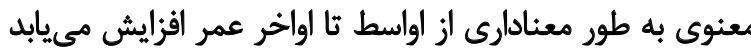

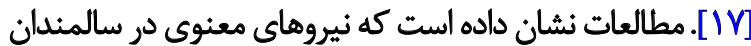

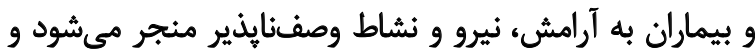

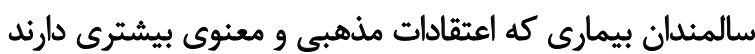

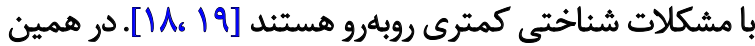

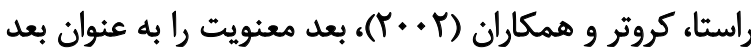

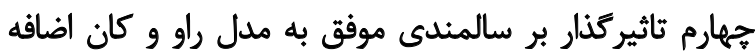

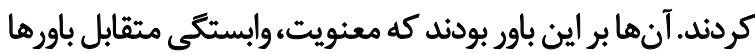

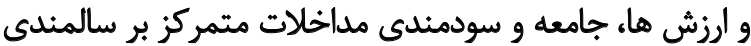

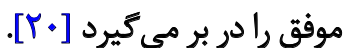

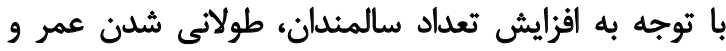

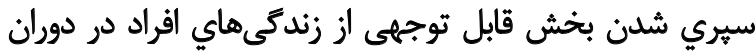

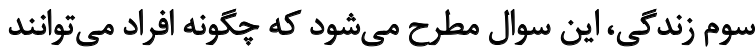
سالمندي موفق داشته باشند. كشور ايران نيز در مسير كذار به به 
نفر) از سالمندان از بيمارىهاى روان رنج مي بردند. مشخصات

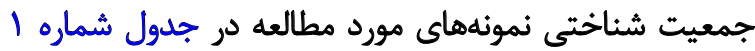

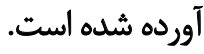

همانطور كه در جدول شماره Y نشان داده شده است، سلامت

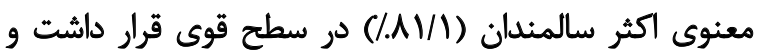

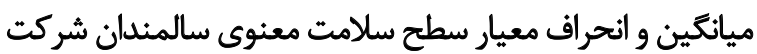

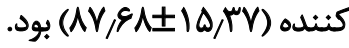

نتايج تحليل ركرسيون به روش استب وايس در جدول شماره

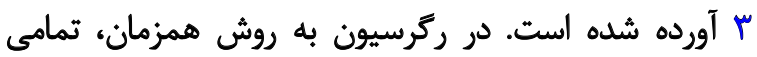

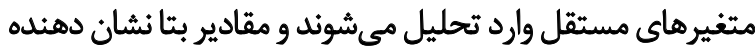

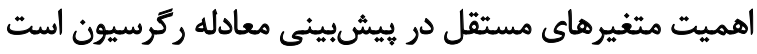

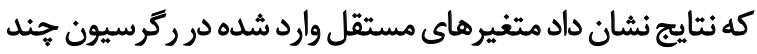

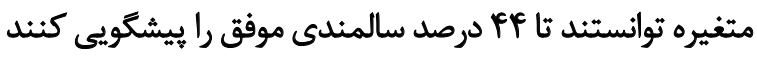

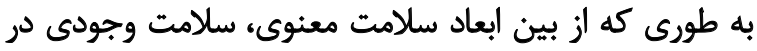

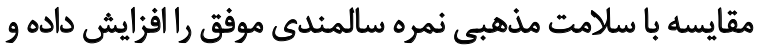

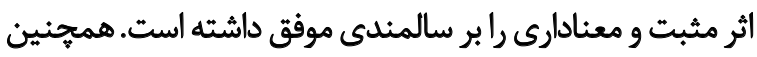

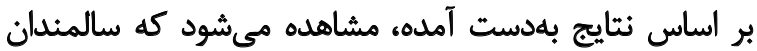

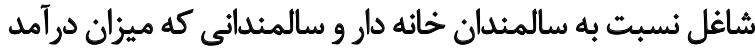
بالايى داشتند در مقايسه با سالمندانى كه ميزاني

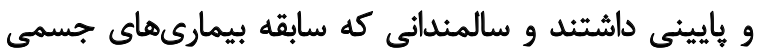

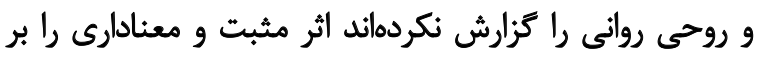

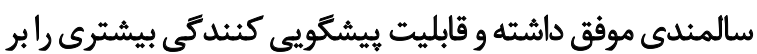

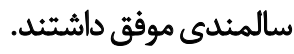

بحث

سالمندي موفق به كسب توانايي بالقوه فردي و سطح بهيئه

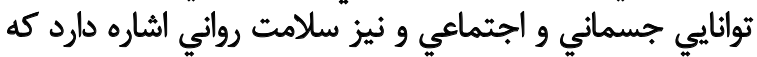

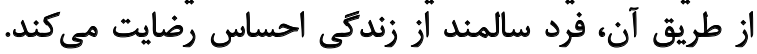

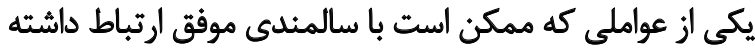

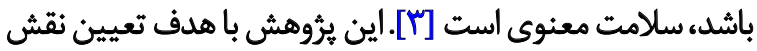

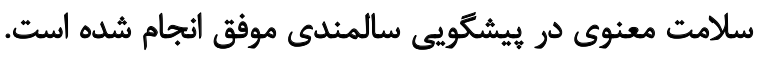

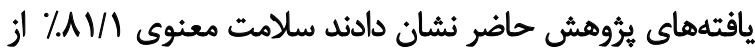

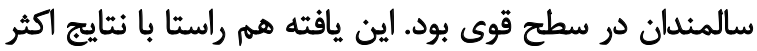

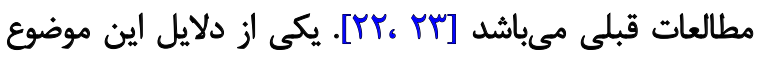

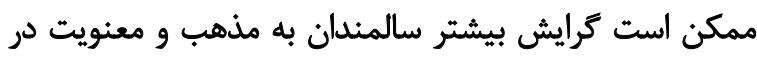

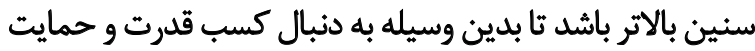

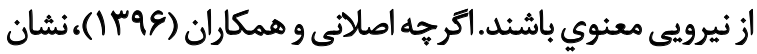

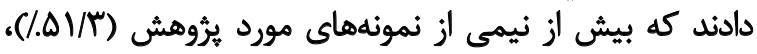

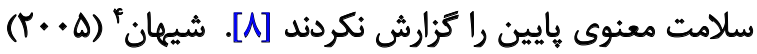

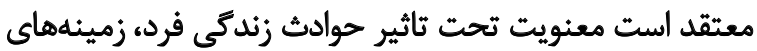

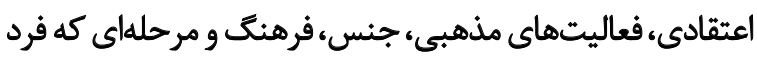

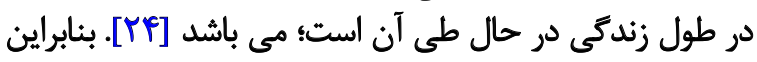

تعيين شاخص روايى محتوا (CVI) يرسشنامه براى • •ا دكترى جواب

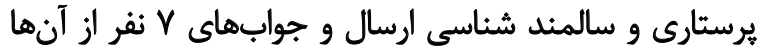

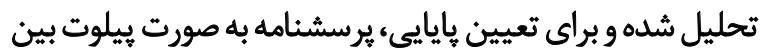

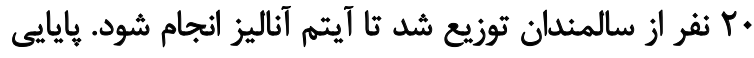

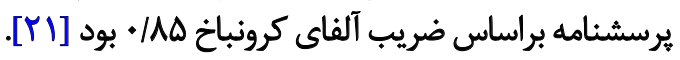

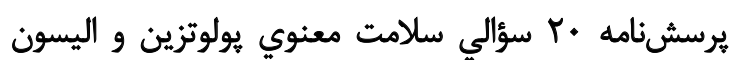

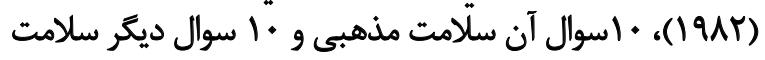

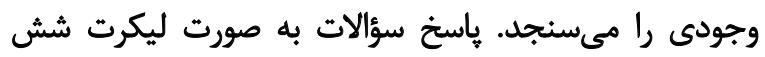

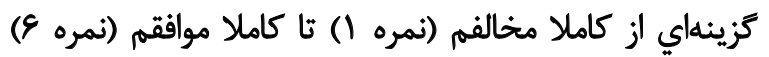

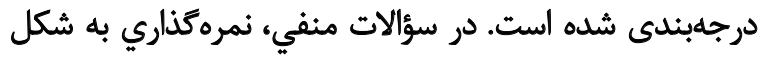

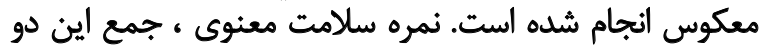

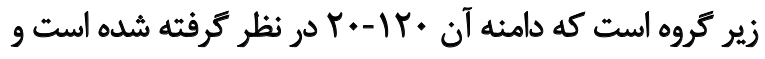

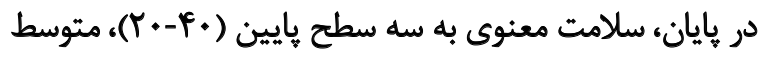

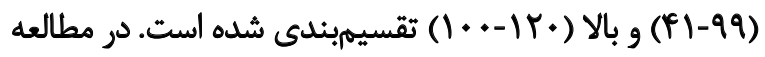

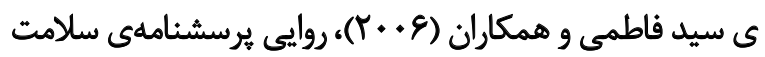

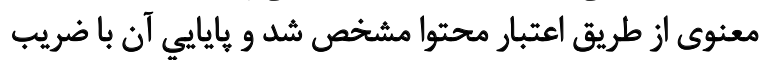
آلفا كرونباخ rA| • مورد تاييد قراركرفت [ • [1]. به منظور رعايت موازين اخلاقى، يس از توضيح اهداف مطالعه

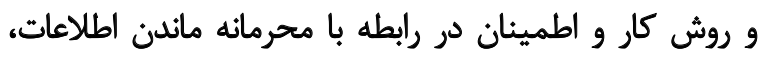

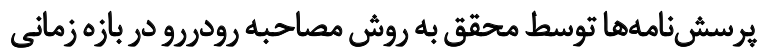

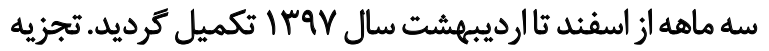

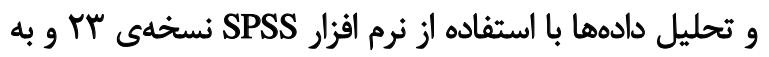

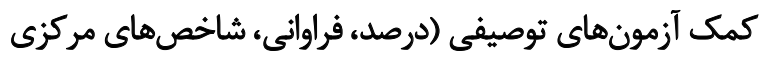

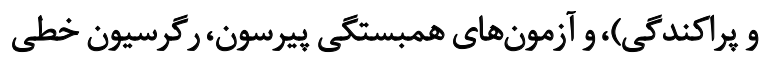

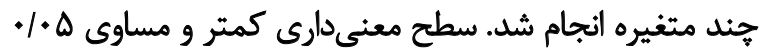
در نظر كرفته شد.

ياقتهها

نمونه مورد مطالعه را | (9 T سالمند با با ميانگين و انحراف معيار

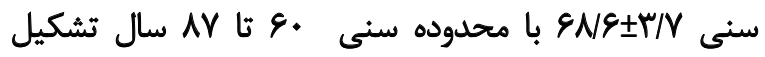

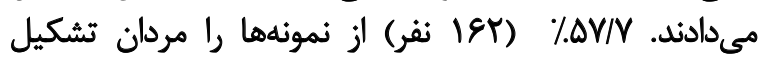

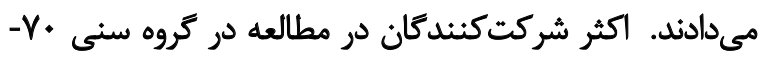

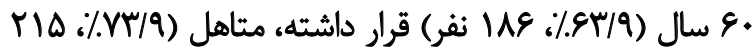

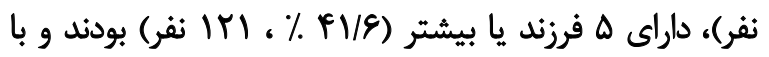

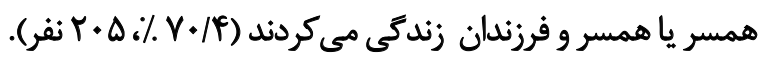

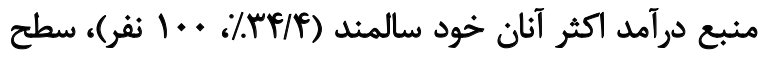

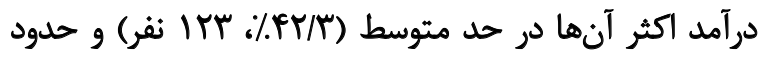

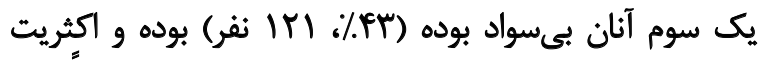

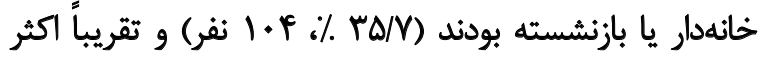

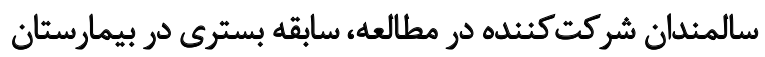

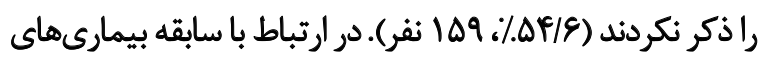

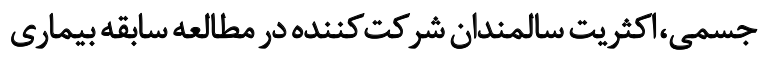

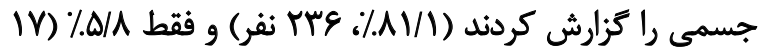


جدول 1. مشخصات جمعيت شناختى سالمندان شركت كننده در ئروهش

\begin{tabular}{|c|c|c|}
\hline فراوانى (درصد) & raw & مثغير \\
\hline $\operatorname{ler}(\Delta \Delta / N)$ & 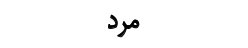 & \multirow{2}{*}{ جنسيت } \\
\hline $\operatorname{lrq}(r e / r)$ & زن & \\
\hline ११ (m4/.) & $8+-8 t$ & \multirow{4}{*}{ سن } \\
\hline AV (rq/q) & $80-89$ & \\
\hline $\operatorname{PV}(I \& / 4)$ & $V \cdot-\eta^{e}$ & \\
\hline$\Delta 1(19 / 9)$ & Vo و بالاتر & \\
\hline$M \otimes\left(M^{m} / 9\right)$ & همسر دار & \multirow{2}{*}{ وضعيت تاهل } \\
\hline$V \&(Y \& /)$ & بلون همسر & \\
\hline$r V(q / Y)$ & بلدون فرزند & \multirow{4}{*}{ تعدا فرزنلان } \\
\hline$p q(1 F / 1)$ & $1-r$ & \\
\hline qf $(M T / Y)$ & $r-\Delta$ & \\
\hline IrI (fI/ & ه هاو بيشتر & \\
\hline $\operatorname{HO}(\mathrm{ET} / \mathrm{H})$ & بي سواد & \multirow{3}{*}{ تحصيلات } \\
\hline $1+\Lambda(r V / N)$ & دييله و كمتر از دييلم & \\
\hline$\Delta 4(19 / 9)$ & أكادميك & \\
\hline $1 . f(r \Delta / V)$ & خانه دار & \multirow{4}{*}{ شغل } \\
\hline$F T(I F / F)$ & بيكار & \\
\hline $1 . p(r \Delta / V)$ & بازتشسشته & \\
\hline Fi (IF/I) & شاغل & \\
\hline $1 . .(M+/ \varphi)$ & خود سالمثل & \multirow{3}{*}{ منبع درآهد } \\
\hline qf $(\pi T / M)$ & خائواده & \\
\hline qr $(M T / M)$ & دولتى & \\
\hline $1 . \Delta(r e / 1)$ & ضعيف & \multirow{4}{*}{ ميزان درآمد } \\
\hline$I T / R T / T)$ & متوسط & \\
\hline$\Delta \cdot(I V / T)$ & خُوب & \\
\hline $\mathbb{I r}(f / \Delta)$ & 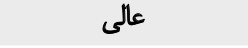 & \\
\hline$r+\Delta(r+/ \varphi)$ & همسر / همسر و فرزندان & \multirow{3}{*}{ نحوه ى زندكى } \\
\hline$M\left(N / /{ }^{\prime}\right)$ & فرزندان & \\
\hline$\Delta \Gamma(W M)$ & تنها & \\
\hline Mre $(1) / 1)$ & 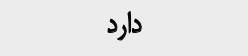 & \multirow{2}{*}{ سابقه ييمارى } \\
\hline$\Delta(W M)$ & تيدارد & \\
\hline $\operatorname{rrg}(A) / 1)$ & دارد & \multirow{2}{*}{ سابقه بسترى } \\
\hline $1 \Delta Q(\Delta F / 8)$ & ندارد & \\
\hline $\operatorname{IV}(\Delta / A)$ & دارد & \multirow{2}{*}{ مشكلات روانى } \\
\hline$T V^{e}(9 q / T)$ & ثلدارد & \\
\hline
\end{tabular}


جدول r. ميزان سلامت معنوى و سطوح آن در سالمندان شركت كنئده در مطالعه

\begin{tabular}{|c|c|c|}
\hline درصد & فراوانى & سلامت معنوى \\
\hline \multicolumn{2}{|c|}{$r(\cdot / M)$} & ضعيف \\
\hline \multicolumn{2}{|c|}{$\Delta M^{\prime}(W T)$} & متوسط \\
\hline \multicolumn{2}{|c|}{$\operatorname{Mrg}(A 1 /)$} & قوى \\
\hline \multicolumn{2}{|c|}{ (9) $(1 . .1 \%)$} & كل \\
\hline محلوده & ميانكين +انحراف معيار & سلامت معنوى \\
\hline$r-119$ & $A V / \& A \pm \backslash Q / T Y$ & r.-IT. \\
\hline
\end{tabular}

جدول r. بيشكُويى سالمئدى موفق براساس ابعاد سلامت معنوى و عوامل دموكرافيك

\begin{tabular}{|c|c|c|c|c|c|c|}
\hline \multicolumn{2}{|c|}{ فاصله اطمينان 90\% } & \multirow{2}{*}{$\mathbf{p}$} & \multirow{2}{*}{ ضريب استاندارد (B) } & \multicolumn{2}{|c|}{ ضرايب غير استائدارد } & \multirow{2}{*}{ متغير } \\
\hline كران بالا & كران بايين & & & أنحراف معيار & بتا & \\
\hline W/e19 & $8 Y / \cdot 11$ & .1. & & $r / 11$ & $V \cdot / M 10$ & ثابث \\
\hline$\cdot|A| \mid$ & $\cdot 10+\Delta$ & $+\ldots$ &.$/ 4 q$. & $+1+\sqrt{r a}$ & $.18 \Delta A$ & سلامت وجودى \\
\hline$-1 / 811$ & $-8 / 19$. & 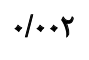 & $-|/| \Delta \mid$ & $1 / 4+9$ & -firaf & (خانه دالم) \\
\hline$N+1$ & IOVY & 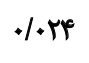 &.$/ 11$ & $1 / 49$ & $p / r q 1$ & (شاغل) \\
\hline - H/ArA & $-1 \cdot / A T^{2}$ & .1. &.$- / T \varepsilon$ & IVA. & - V/MTr & (ضعيف) \\
\hline.$- / 1$ fif & $-g / M+4$ & $. \mid+4)$ &.$- / 178$ & Veve & $-r / p p f$ & (sتوسط) \\
\hline -VPIT & -IV/AV. & $.1 \%$ &.$- /$ MIV & $T / V \cdot V$ & $-Y(\Delta P)$ & مشكلات روحى (خير) \\
\hline V/\&AY & I/FTA & $+1+*$ &.$/ I M T$ & $1 / 09$. & F/DAF & سابقه بيمارى (خير) \\
\hline
\end{tabular}

Model summary R=0.633, R² =0.440, Adjusted R2 =0.424. SE: Standard Error, Cl: Confidence Interval

طرفي نتايج مطالعه حاضر نشان داد از ميان ابعاد سلامت معنوى،

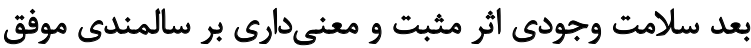

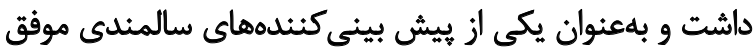

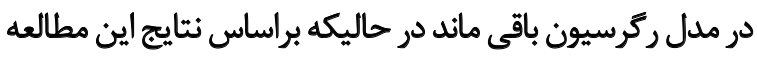

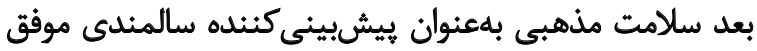

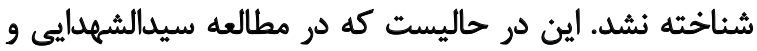

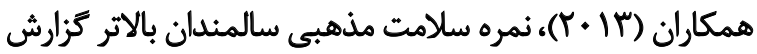

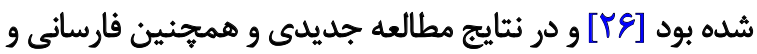

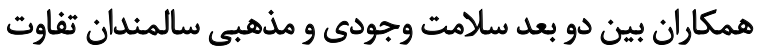

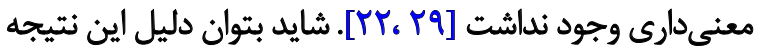

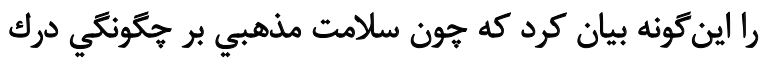

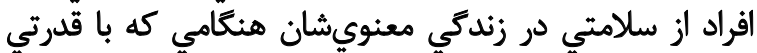

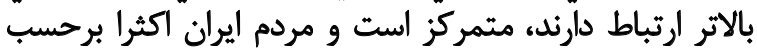

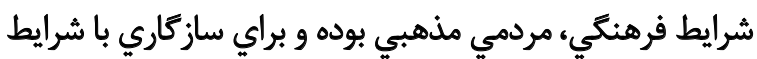

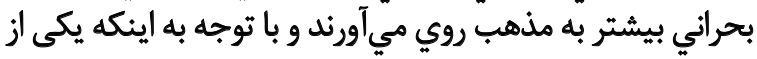

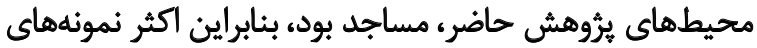

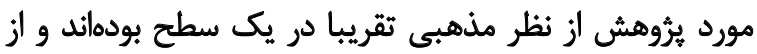

سطح سلامت معنوى متفاوت سالمندان مي تواند از تفاوت در

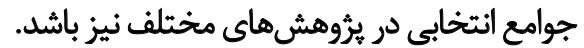
يافتههاي اين يرؤوهش نشان دادند كه بين سلامت معنوى و

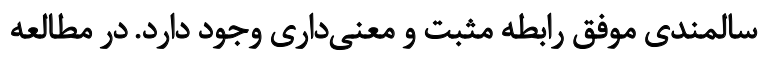

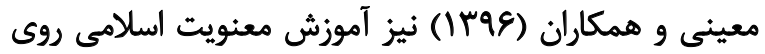

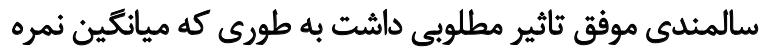

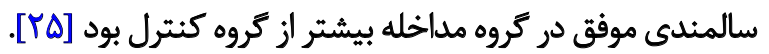

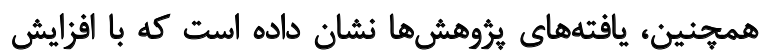

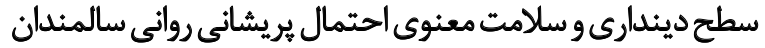

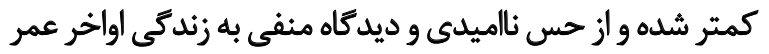

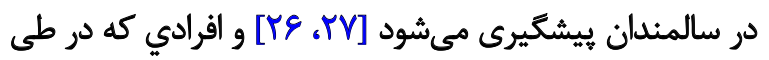

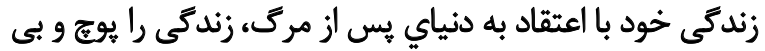

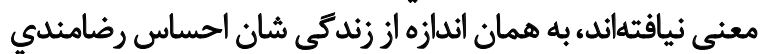

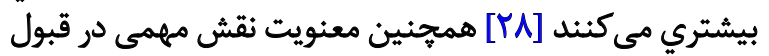

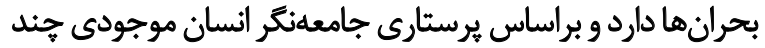

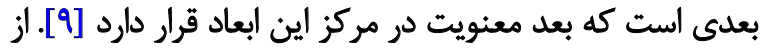


طي كنند، برنامههاى آموزش معنويت و بهبود سلامت وجودي

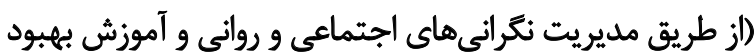

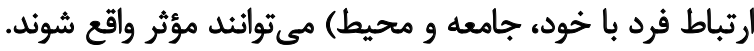

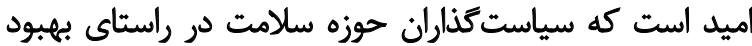
معنويت در سالمندان اقدامات عملى موثرى ران رانجام دهئد از محدوديتهاى مطالعه حاضر ميىوان به استفاده از

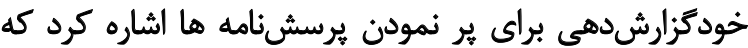

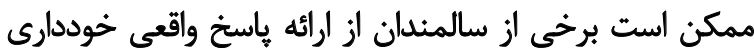

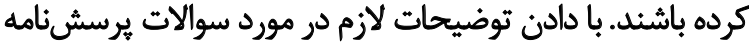

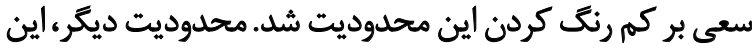

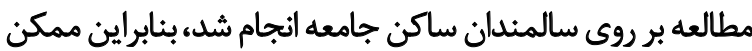

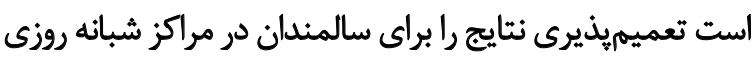

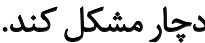

\section{مالاحظات اخلاقى}

\section{يبروى أز اصول الخلاق يُوهش}

اصول اخلاقى تماماً در اين مقاله رعايت شده است. شركت

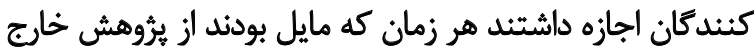

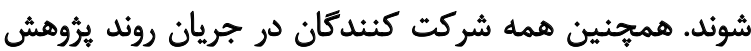
بودند. اطلاعات آن ها محرمانه نكّه داشته شد شد.

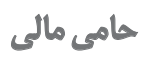

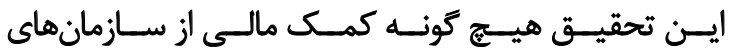

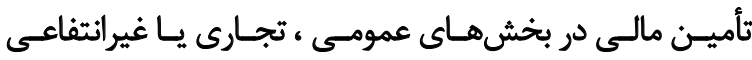

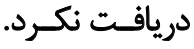

$$
\text { مشاركت نويسئد مَان }
$$

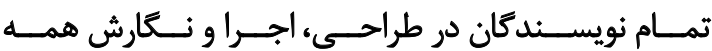

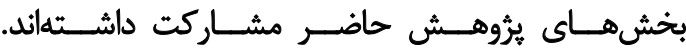

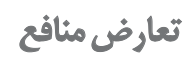

بنابر اظهار نويسندكان، اين مقاله تعارض منافع ندارد.

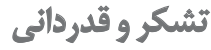

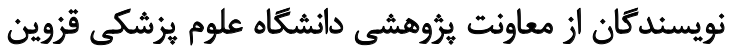

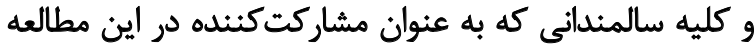

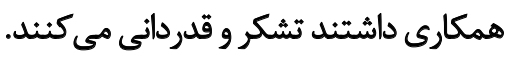

طرفي در دوران سالمندى به علت افزايش مشكلات و بيمارىهاي

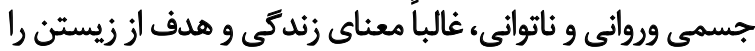

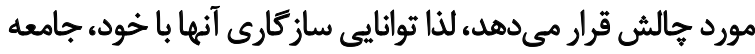

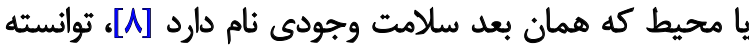

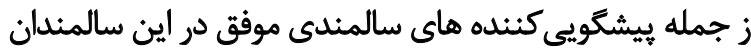
باشد.

نتايج اين مطالعه نشان دادند از بين متغيرهاى دموكرافيك

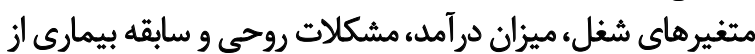

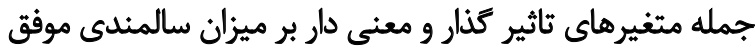

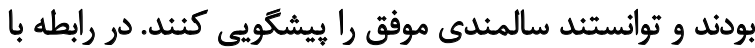

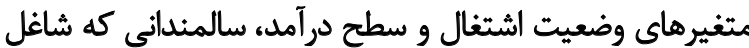

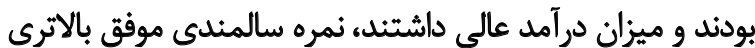

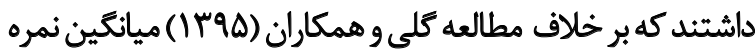

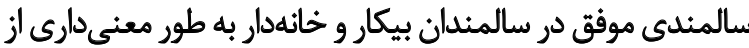

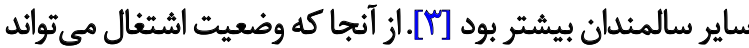

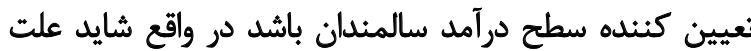

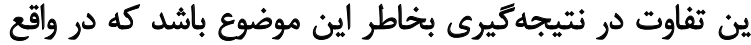

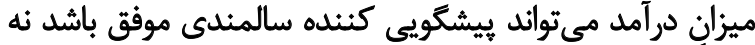

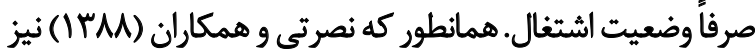

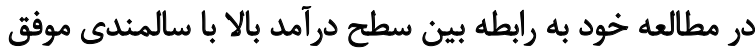

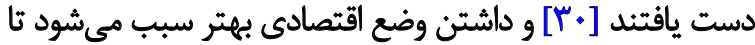

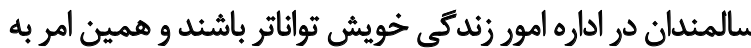

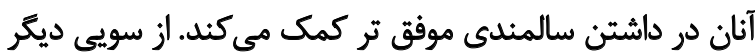

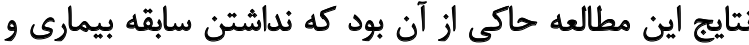

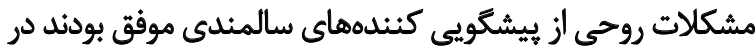

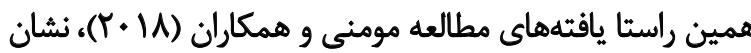

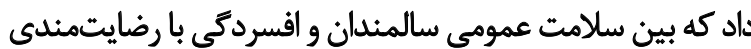

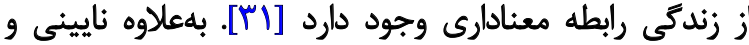

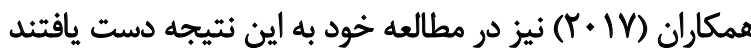

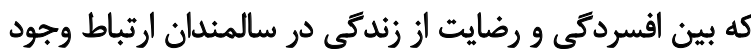

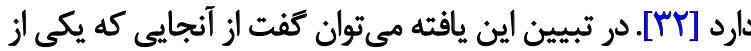

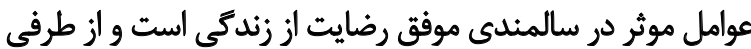

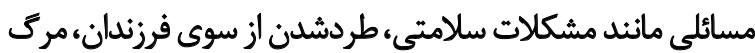

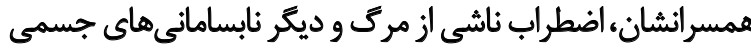

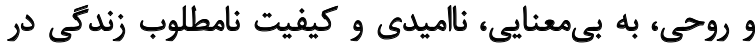

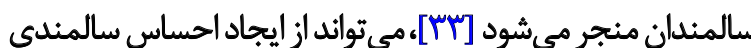

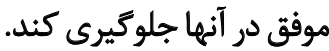

\section{نتيجلكَيرى نهمايى}

نتايج اين ثئوهش نشان دادند كه بين سلامت معنوى و سالمندى

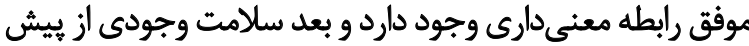

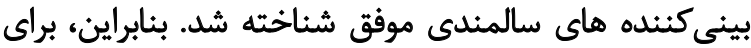

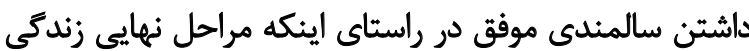

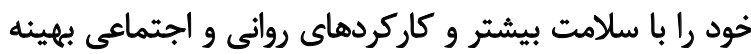




\section{References}

[1] Hashemlou L, Hemati Maslak Pak M, Khalkhali H. [Effect of Orem self care program for the elderly self-care ability (Persian)]. Journal of Urmia Nursing and Midwifery Faculty. 2014; 11(2):11926. http:/ / unmf.umsu.ac.ir/article-1-1245-fa.html

[2] Alipour F, Sajadi H, Setare F, Beyglorian A, Jalileans A. [Elderly Quality of Life in Tehran's District Two (Persian)]. Iranian Journal of Aging. 2007; 10(3):72-80. http://salmandj.uswr.ac.ir/article1-104-fa.html

[3] Goli M, Maddah S, Dalvandi A, Hosseini M, Rahgozar M, Mousavi Arfa N. [The Relationship between successful aging and spiritual health of the elderly (Persian)]. Iranian Journal of Psychiatric Nursing. 2016; 4(5):16-21. [DOI:10.21859/ijpn-04053]

[4] Miri N, Maddah M, Raghfar H. [Aging and Economic Growth (Persian)]. Salmand: Iranian Journal of Ageing. 2019; 13 (5):626637 [DOI:10.32598/SIJA.13.Special-Issue.626]

[5] Larkin M, Butler R. Championing a healthy view of ageing. Lancet. 2001; 357(95):48-9. [DOI:10.1016/S0140-6736(05)71550-6]

[6] Motamedi A. The role of life events in successful aging. Journal of Social Sciences and Humanities Shiraz University. 2005; 22(4):189203. https:// www.sid.ir/en/journal/ViewPaper.aspx?ID=51132

[7] Stephens C, Flick U. Health and ageing--challenges for health psychology research. Journal of Health Psychology. 2010; 15(5):643-8. [DOI:10.1177/1359105310368178] [PMID]

[8] Aslani Y, Hosseini R, Alijanpour-Aghamaleki M, JavanbakhtianGhahfarokhi R, Borhaninejad V. [Spiritual health and life satisfaction in older adults in Shahrekord hospitals (Persian)]. Journal of Clinical Nursing and Midwifery. 2017; 6(4):1-10. http://jcnm. skums.ac.ir/article-1-609-fa.html

[9] Sodagar S, Sobhi N. [The distinctive role of spirituality and social support in mental health and death anxiety in the elderly (Persian)]. Social Psychology Research. 2019; 8(29):1-22. http:// www.socialpsychology.ir/article_80818_aaefb60b5475c0e2d8a9068104fc459e.pdf?lang=en

[10] Seyed Fatemi N, Rezaei M, Givari A, Hosseini F. Prayer and spiritual well-being in cancer patients. Payesh. 2006; 5(4):295-303. https:// www.sid.ir/en/Journal/ViewPaper.aspx?ID=69936

[11] Gonzalez P, Castaneda S, Dale J, Medeiros E, Buelna C, Nunez $\mathrm{A}$, et al. Spiritual well-being and depressive symptoms among cancer survivors. Supportive Care in Cancer. 2014; 22(9):2393-400. [DOI:10.1007/s00520-014-2207-2] [PMID] [PMCID]

[12] Daaleman T, Dobbs D. Religiosity, spirituality and death attitudes in chronically III older adults. Research on Aging. 2010; 32(2):224-43. [DOI:10.1177/0164027509351476]

[13] McCain N. Psychoneuroimmunology, spirituality, and cancer. Gynecologic Oncology. 2005; 99(3):121. [DOI:10.1016/j.ygyno.2005.07.055] [PMID]

[14] Brown D, Johnson E, Parrish M. Spirituality assessments: Limitations and recommendations [Internet]. 2007 [Updated 2007]. Available from: https:/ / wwwcounselingorg/resources/library/ vistas/ 2007-V-online-MSWord-files/2007-V-online-MSWordfiles/Browndoc. 2007

[15] Musarezaie A, Kadkhodaei-Elyaderani H, Ghasemipoor M, Havayi A, Darvish M. [The predictive role of psychological, medical and demographic factors, on spiritual well-being in patients with breast cancer (Persian)]. International Journal of Behavioral Sciences. 2015; 13(2):266-75. http://rbs.mui.ac.ir/ article-1-402-fa.html
[16] Lavretsky H. Spirituality and aging. Aging Health. 2010; 6(6):749-69. [DOI:10.2217/ahe.10.70]

[17] Wink P, Dillon M. Spiritual development across the adult life course: Findings from a longitudinal study. Journal of Adult Development. 2002; 9(1):79-94. [DOI:10.1023/A:1013833419122]

[18] Kazemi N, Sajjadi H, Bahrami G. [Quality of life in Iranian elderly (Persian)]. Salmand: Iranian Journal of Ageing. 2019; 13(5):518-33. [DOI:10.32598/SIJA.13.Special-Issue.518]

[19] Khoshbakht Pishkhani M, Mohammadi Shahboulaghi F, Khankeh H, Dalvandi A. [Spiritual health in Iranian elderly: A concept analysis by walker and avant's approach (Persian)]. Salmand. Iranian Journal of Ageing. 2019; 14(1):96-113. http://salmandj.uswr.ac.ir/article-1-1424-fa.html

[20] Crowther M, Parker M, Achenbaum W, Larimore W, Koenig H. Rowe and Kahn's Model of successful aging revisited: Positive spirituality-the forgotten factor. The Gerontologist. 2002; 42(5):613-20. [DOI:10.1093/geront/42.5.613] [PMID]

[21] Habibi A, Nikpour S, Seiedoshohadaei M, Haghani H. [Quality of life and status of physical functioning among elderly people in west region of Tehran: A Cross-Sectional Survey (Persian)]. Iran Journal of Nursing. 2008; 21(53):29-39. http://ijn.iums.ac.ir/ article-1-406-fa.html

[22] Jadidi A, Farahaninia M, Janmohammadi S, Haghani H. [Spiritual well being of elderly people resident in nursing home (Persian)]. Journal of Geriatric Nursing. 2015; 1(2):22-30. http://jgn. medilam.ac.ir/article-1-74-fa.html

[23] Rezaie Shahsavarloo Z, Lotfi M, Taghadosi M, Mousavi M, Yousefi Z, Amirkhosravi N. [Relationship between components of Spiritual well-being with hope and life satisfaction in elderly cancer patients in Kashan (Persian)]. Iranian Journal of Geriatric Nursing. 2015; 1(2):43-54. http://jgn.medilam.ac.ir/article1-76-fa.html

[24] Sheehan M. Spirituality and the care of people with life-threatening illnesses. Techniques in Regional Anesthesia and Pain Management. 2005; 9(3):109-13. [DOI:10.1053/j.trap.2005.06.002]

[25] Moeini M, Sharifi S, Zandiyeh Z. Does Islamic spiritual program lead to successful aging? A randomized clinical. Journal of Education and Health Promotion. 2016; 5:2. [DOI:10.4103/22779531.184561] [PMID] [PMCID]

[26] Saydshohadai M, Haghani H, Heshmat S, Seidfatemi N, Mehrdad N. [The Spiritual Health of Seniors Living in Sanitarium and Home Residents (Persian)]. Iran Journal of Nursing (IJN). 2013; 26(81):11-20. http://ijn.iums.ac.ir/article-1-1509-fa.html

[27] Sodagar S, Sobhi S. [The distinctive role of spirituality and social support in mental health and death anxiety in the elderly (Persian)]. Social Psychology Research. 2019; 8(29):1-29. http:// www.socialpsychology.ir/article_80818.html

[28] Mo'tamedi A, Doostian U, Jalalvand M, Farzanwgan M. The Role of Resiliency, Spirituality, and Religiosity in Predicting Satisfaction with Life in the Elderly. Quarterly Journal of Counseling and Psychotherapy. 2012; 3(12):1-20. [DOI:10.22054/QCCPC.2011.5906]

[29] Farsani M, Alavi M, Parvizi S, Haghani H. [Study of spiritual health and quality of life in multiple sclerosis patients admitted to the MS Society of Iran (Persian)]. [MSc. thesis]. Tehran: Tehran University; 2008. http://dspace.tbzmed.ac.ir:8080/xmlui/handle/123456789/20422. 
[30] Momeni K, Rafiee Z. [Correlation of Social Support and Religious Orientation With Life Satisfaction in the Elderly (Persian)]. Salmand: Iranian Journal of Ageing. 2018; 13(1):50-61. [DOI:10.21859/sija.13.1.50]

[31] Emaminaeini M, Bakhtiyari M, Hatami H, Khodakarim S, Sahaf R. [Elderly the in Support Social Perceived and Depression (Persian)]. Iranian Journal of Aging. 2017; 12(2):192-207. [DOI:10.21859/sija-1202192]

[32] Saffarinia M, Dortaj A. [Effect of Group Logotherapy on Life Expectancy and Mental and Social Wellbeing of The Female Elderly Residents of Nursing Homes in Dubai (Persian)]. Salmand: Iranian Journal of Ageing. 2018; 12 (4):482-93. http://salmandj. uswr.ac.ir/article-1-1319-fa.html 
This Page Intentionally Left Blank 